Wetzels, Peter. Zur Nieden, Valentin, “Confesiones falsas - Frecuencias mundiales e implicaciones de la investigación", Nuevo Foro Penal 95, (2020).

\title{
Confesiones falsas - Frecuencias mundiales e implicaciones de la investigación
}

\section{"False Confessions - Worldwide Frequencies and Research Implications"}

Prof. Dr. Peter Wetzels**

Valentin ZUR NIEDEN ${ }^{*}$

Fecha de recepción: 30/06/2020. Fecha de aceptación: 27/11/2020

DOI: $10.17230 / n f p 16.95 .1$

\section{Resumen}

Las investigaciones realizadas en las últimas tres décadas han demostrado la existencia de confesiones falsas en los interrogatorios policiales que se producen por todo el mundo. Asimismo, las razones y las circunstancias de las confesiones falsas también han sido investigadas. No obstante, la frecuencia de este fenómeno aún se desconoce en gran medida. Existen diversos estudios, que han abordado este tema. Pero, la naturaleza de una confesión falsa hace que sea difícil su reconocimiento, al igual que recopilar datos verídicos sobre su frecuencia. La comparación de diversos estudios muestra, que las confesiones falsas ocurren mucho más a menudo de lo que se piensa. Este artículo presenta el estado actual de la investigación sobre la frecuencia de las confesiones falsas y discute los enfoques de investigaciones realizadas.

*Estudiante de doctorado en la Universidad de Hamburgo, contacto: mail@zur-nieden.de. El texto es uno de los resultados de la investigación para la tesis doctoral.

**Profesor de Criminología de la Universidad de Hamburgo, supervisor del doctorado, contacto: peter.wetzels@uni-hamburg.de. 


\section{Abstract}

Research over the past three decades has shown that false confessions exist in police interrogations around the world and that they appear in different forms. The reasons and circumstances of false confessions have also been investigated. However, the frequency of this phenomenon is still largely unknown. Although many studies have addressed this issue, the nature of false confessions makes it difficult to recognize them, as well as to collect reliable data on their frequency. A comparison of different studies shows, however, that false confessions occur far more often than one might think. This article presents the current state of research on the frequency of false confessions and discusses the research approaches taken.

\section{Palabras clave}

Confesiones falsas, interrogatorios policiales, acusados, detenidos.

\section{Keywords}

False confessions, police interrogations, defendants, detainees.

\section{Sumario}

1. Introducción; 2.La Temática; 3.Frecuencia de las confesiones falsas;

4.Conclusiones; 5.Bibliografía.

\section{Introducción}

Durante siglos, la confesión fue la "reina de la evidencia". ${ }^{1}$ Para obtenerla, se utilizaban medidas drásticas, como la tortura. Incluso, en la época del Imperio Romano, las confesiones hechas bajo tortura fueron llamadas "la forma más pura de la verdad". ${ }^{2}$ Sólo a partir del siglo XVIII, con el inicio de la llustración en Europa, se concedió un mayor enfásis en la evidencia; que permitió un cambio en la importancia de la

1 Edward Schramm. "Das Geständnis im Deutschen Strafprozess", En Das Geständnis und seine Instanzen: Zur Bedeutungsverschiebung des Geständnisses im Prozess der Moderne, editado por Anders Engberg-Pedersen; Michael Huffmaster; Eric Nordhausen; Vrääth Öhner. (Berlin: Turia + Kant, 2011), 46; compárese, también a lo siguiente: Valentin zur Nieden. "Falsche Geständnisse in der polizeilichen Beschuldigtenvernehmung. Eine interdisziplinäre Darstellung der theoretischen Grundlagen falscher Geständnisse und zwei empirische Untersuchungen von Polizeibeamten und Inhaftierten zur Häufigkeit und den Gründen von falschen Geständnissen in Deutschland". (tesis doctoral todavía inédita de la Universidad de Hamburgo, 2020).

2 Gavin Oxburgh, Ivar Fahsing, Kate Haworth y Pete Blair "Interviewing Suspected Offenders", En Communication in Investigative and Legal Contexts: Integrated Approaches from Forensic Psychology, Linguistics and Law Enforcement, 1. Edición, editado por Gavin Oxburgh; Trond Myklebust; Tim Grant; Rebecca Milne. (Chichster: Wiley-Blackwell, 2015), 136. 
confesión, ya que pasó de ser la pieza más importante en una investigación a ser una pieza más de la evidencia. Asimismo, hubo cambios en el interrogatorio policial, por ejemplo; los métodos de intimidación y coerción que una vez prevalecieron fueron reemplazados por enfoques cada vez más cooperativos. No obstante, este desarrollo no pudo suprimir por completo la importancia de la confesión. De hecho, hoy en día, muchos sospechosos, tanto en los interrogatorios policiales como en los procedimientos judiciales son condenados en base a sus confesiones.

Las estimaciones criminológicas arrojan que las confesiones suponen entre un 60 a 80 por cierto de los casos en una investigación policial. ${ }^{3}$ Teniendo en cuenta estos números, es comprensible que Gunter Arzt considere que el sistema criminal actual se derrumbaría si no fuera por un alto porcentaje de perpetradores que confiesan ${ }^{4}$. Hay que mencionar, además que la confesión sigue siendo el objetivo de cualquier trabajo de investigación. ${ }^{5}$ Pues desde un punto de vista económico, cada confesión supone un gran éxito. Por un lado, los investigadores pueden buscar información en la confesión. Por otro lado, el juez puede resolver hechos confusos con una referencia al contenido de la confesión ${ }^{6}$. Finalmente, el acusado tiene la ventaja de alegar circunstancias atenuantes.

Respecto a lo anterior, la confesión puede convertirse en un „lugar de complicidad silenciosa entre todas las instancias de justicia penal". Sin embargo, a partir de esta situación ventajosa para todos los involucrados, si se da una confesión falsa, pueden surgir rápidamente desventajas importantes para el acusado. Por consiguiente, existe un gran peligro en el fenómeno, conocido como el „poder mágico de la confesión"7: la idea de que el perpetrador ha confesado los hechos conlleva a que no se verifiquen los mismos y las contradicciones ya no tengan importancia. Así pues, si un sospechoso realiza una confesión falsa por agotamiento en un interrogatorio al que fue sometido con presión, y tiene la esperanza de que la policía reconozca

3 ZUR NIEDEN, "Falsche Geständnisse in der polizeilichen Beschuldigtenvernehmung. Eine interdisziplinäre Darstellung der theoretischen Grundlagen falscher Geständnisse und zwei empirische Untersuchungen von Polizeibeamten und Inhaftierten zur Häufigkeit und den Gründen von falschen Geständnissen in Deutschland."

4 Schramm, "Das Geständnis im Deutschen Strafprozess", 34.

5 Ibíd., 34, 46.

6 Compárese, también a lo siguiente: VRÄÄTH Öhner. "Unabweisbare Gewissheit. Zur Kritik des Geständnisses", En Das Geständnis und seine Instanzen: Zur Bedeutungsverschiebung des Geständnisses im Prozess der Moderne, editado por Anders Engberg-Pedersen; Michael Huffmaster; Eric Nordhausen; Vrääth Öhner. (Berlin: Turia + Kant, 2011), 29.

7 Ibíd, 30. 
automáticamente la falsedad de ésta mediante investigaciones adicionales, esa esperanza se puede ver amargamente decepcionada, ya que la la policía, en el caso seguro de tener al delincuente correcto, no investiga (lo suficiente) y la falsedad de la confesión no se revela.

Dicha confesión falsa según la clasificación de Kassin y Wrightsman, ${ }^{8}$ se denomina como confesión falsa forzada conforme ("coerced-compliant"). Tal confesión, se hace deliberadamente para lograr un objetivo específico, tal como, finalizar el interrogatorio. ${ }^{9}$

Según Kassin y Wrightsman, los otros dos tipos de confesiones falsas se denominan confesiones falsas forzadas internalizadas ("coerced-internalized") y voluntarias ("voluntary false confessions"). El primer tipo se da, cuando el sospechoso está tan sugestivamente influenciado por los funcionarios que cree que cometió el crimen, aunque en realidad no lo haya perpetrado. Por lo tanto, sin saberlo, se culpa por algo que no hizo. ${ }^{10}$ Por otro lado, el segundo tipo se da, cuando alguien admite un acto sin haber sido sospechoso y sin la intervención de las autoridades investigadoras. ${ }^{11}$ Pueden ser varias las razones para llegar a dicha situación: a menudo, el confesor quiere cubrir al presunto o verdadero delincuente ${ }^{12}$ y en otros casos, se sospecha una necesidad patológica de atraer atención 0 una pérdida de la realidad. ${ }^{13}$

\section{La temática}

El ejemplo más conocido de confesiones falsas es el caso de los cinco adolescentes que fueron conocidos como los "Central Park Five”. En 1989, una deportista fue violada, golpeada y dejada moribunda en el Central Park de Nueva

8 Compárese, también a lo siguiente: Saul Kassin y Lawrence Wrightsman. "Confession Evidence", En The Psychology of Evidence and Trial Procedure, editado por Saul Kassin; Lawrence Wrightsman. (Beverly Hills: Sage, 1985), 76; Lawrence Wrightsman y Saul Kassin. Confessions in the Courtroom. (London: Sage, 1993), 92; Renate Volbert. "Falsche Geständnisse bei Kapitaldelikten. Grundlagen", En Festschrift für Ulrich Eisenberg zum 70. Geburtstag, editado por Henning Müller; Günther Sander; Helena Válková. (München: Beck, 2009), 69.

9 Renate Volbert, Claudia Böhm y Falsche Geständnisse", En Handbuch der Rechtspsychologie, editado por Renate Volbert; Max Steller. (Göttingen: Hgrefe, 2008), 254.

10 Ibíd., 254.

11 Ibíd., 253.

12 Ibíd., 254.

13 Saul Kassin y Gisli Gudjonsson. "The Psychology of Confessions. A Review of the Literature and Issues", Psychological Science in the Public Interest, $5^{a}$ época, n² 2, (2004): 33. 
York ${ }^{14}$. Después de intensos interrogatorios y pasadas las 72 horas del crimen, los cinco adolescentes, de entre 14 y 16 años, confesaron los hechos. Basándose solamente en sus confesiones verbales, que fueron detalladas, pero también contradictorias y defectuosas, los sospechosos fueron condenados a largas penas de prisión. Casi nadie dudaba de su culpabilidad, aún cuando no había más pruebas en su contra. Incluso las pruebas de ADN realizadas con rastros de esperma en la víctima excluyeron a todos como perpetradores. Pasaron13 años después para que el verdadero perpetrador, Matias Reyes, un prisionero condenado por dos violaciones y un asesinato, confesara el crimen. Se descubrió que tenía un conocimiento exclusivo del delito y que había dejado rastros de ADN en la escena del crimen. Poco tiempo después, los cinco inocentes fueron absueltos.

En base a este drástico caso, entre otros, la temática de las confesiones falsas, particularmente los interrogatorios policiales, se han convertido cada vez en el centro de investigación internacional en los últimos años. El hecho de que las personas hagan confesiones falsas ha sido probado internacionalmente, ${ }^{15}$ al igual que la pregunta de por qué se hacen tales declaraciones. En cambio, la cuestión relevante es conocer la frecuencia con la que se producen las confesiones falsas ${ }^{16}$. Actualmente, esta cuestión sigue sin resolverse y es que, encontrar solución a ello no es fácil, inclusive puede llegara ser imposible por varias razones. ${ }^{17}$

En el caso de realizar deliberadamente la confesión de manera incorrecta, lo que persigue el confesor es que no se descubra la falsedad de su confesión. En ocasiones, esto puede deberse al hecho de que una tercera persona esté siendo protegida. ${ }^{18}$ Por otro lado, las confesiones inconscientes, y las que son resultado de

14 Saul Kassin. "The social psychology of false confessions", Social Issues and Policy Review, ga época, $n^{0} 1,(2015): 25$.

15 Renate Volbert, Lennart May, Joachim Hausam y Steffen Lau. "Confessions and Denials When Guilty and Innocent: Forensic Patients' Self-Reported Behavior During Police Interviews", Frontiers in Psychiarty, 10ª́poca, nº 168, (2019): 1.

16 ZUR NiedEN, "Falsche Geständnisse in der polizeilichen Beschuldigtenvernehmung. Eine interdisziplinäre Darstellung der theoretischen Grundlagen falscher Geständnisse und zwei empirische Untersuchungen von Polizeibeamten und Inhaftierten zur Häufigkeit und den Gründen von falschen Geständnissen in Deutschland."

17 Volbert, Lennart, Hausam y Lau, "Confessions and Denials When Guilty and Innocent: Forensic Patients' Self-Reported Behavior During Police Interviews", 1.

18 ZUR NIEDEN, "Falsche Geständnisse in der polizeilichen Beschuldigtenvernehmung. Eine interdisziplinäre Darstellung der theoretischen Grundlagen falscher Geständnisse und zwei empirische Untersuchungen von Polizeibeamten und Inhaftierten zur Häufigkeit und den Gründen von falschen Geständnissen in Deutschland." 
la presión policial (no intencionalmente, pero sí conscientemente), tienen el problema común de la falta de un criterio externo que pueda probar una confesión falsa como tal. ${ }^{19}$ En ocasiones, estas confesiones falsas solo se descubren si se encuentra al verdadero perpetrador. ${ }^{20}$

No existen estadísticas oficiales ni estimaciones representativas confiables de la frecuencia de las confesiones falsas, ${ }^{21}$ por lo que la frecuencia de este fenómeno solo puede aproximarse combinando diferentes enfoques. Para obtener estos valores aproximados, se realizan principalmente: análisis de archivos, investigaciones experimentales y encuestas cuantitativas. El siguiente texto muestra el estado actual de la investigación internacional sobre la frecuencia de las confesiones falsas, la evalúa y muestra nuevas necesidades de investigación.

\section{Frecuencia de las confesiones falsas}

\subsection{Frecuencia en los análisis de archivos}

En particular, los estudios estadounidenses muestran que las confesiones falsas no son un fenómeno marginal. ${ }^{22} \mathrm{~A}$ pesar de que los valores diferen, los resultados de Bedau y Radelet ${ }^{23}$ (14\% confesiones falsas), Gross et al. ${ }^{24}(15 \%)$, Rattne ${ }^{25}$ $(8,4 \%)$, Connors et al. ${ }^{26}(18 \%)$ y Scheck et al. ${ }^{27}(24 \%)$ muestran que las confesiones

19 Volbert, 207.

20 Regina Lange. Fehlerquellen im Ermittlungsverfahren. Eine Auswertung von 1110 Wiederaufnahmeverfahren. (Heidelberg: Kriminalistik, 1980), 90.

21 ZUR Nieden, "Falsche Geständnisse in der polizeilichen Beschuldigtenvernehmung. Eine interdisziplinäre Darstellung der theoretischen Grundlagen falscher Geständnisse und zwei empirische Untersuchungen von Polizeibeamten und Inhaftierten zur Häufigkeit und den Gründen von falschen Geständnissen in Deutschland."

22 Ibíd.

23 Hugo Bedau y Michael Radelet. "Miscarriages of Justice in Potentially Capital Cases", Stanford Law Review, n0 40, (1987), 57.

24 Samuel Gross, Kristen Jacoby, Daniel Matheson, Nicholas Montgomery y Sujata Patll. "Exonerations in the United States 1989 through 2003", Journal of Criminal Law and Criminology, 95a época, n², (2005), 544.

25 Arye Rattner. "Convicted but innocent: Wrongful conviction and the criminal justice system", Law and Human Behavior, 12ª́poca, nº 3, (1988): 291.

26 Edward Connors, Thomas Lundregan, Neal Miller y Tom McEwen. Convicted by Juries, Exonerated by Science. Case Studies in the Use of DNA Evidence to Establish Innocence After Trial. (Alejandría: Departamento de Justicia de los EE.UU., Oficina de Programas de Justicia, Instituto Nacional de Justicia, 1996), 16. 
falsas se producen en una medida considerable. Estos resultados son confirmados por las evaluaciones del Proyecto Inocencia ${ }^{28}(29 \%)$ y del Registro Nacional de Exoneraciones $^{29}(12,2 \%)$.

En Alemania, en el año 1965, Kiwit ${ }^{30}$ encontró solo un 2\% de confesiones falsas, y en el análisis realizado por Peters, de más de 1.000 juicios entre los años 50 y $60^{31}$, se encontró una proporción de confesiones falsas del 7\%. Por otra parte, en un estudio reciente, Dunke| ${ }^{32}$ llevó a cabo un análisis de archivos de 48 juicios de la ciudad hanseática de Hamburgo entre 2003 y 2015, pero no encontró ninguna confesión falsa. Leuschner et al., ${ }^{33}$ examinaron en 2019 todos los casos alemanes accesibles de juicios exitosos entre 1990 y 2016, identificando 29 casos con un total de 31 personas condenadas y encarceladas por error, y en cinco casos la condena se basó en una confesión falsa (16\%).

Se puede ver que un número considerable de confesiones falsas fueron probadas en muchos estudios. Es probable que las cifras que se presentan en este trabajo también sean particularmente sólidas, ya que se trata de casos que están respaldados por archivos. Sin embargo, hay que tener en cuenta que, por su propia naturaleza, los análisis de los archivos solo pueden informar de confesiones falsas que también se hayan registrado en archivos.

28 https://www.innocenceproject.org/all-cases/\#false-confessions-or-admissions, exon erated-by-dna

29 http://www.law.umich.edu/special/exoneration/Pages/ExonerationsContribFactorsBy Crime.aspx

30 Walter Kıwit. Fehlurteile im Strafrecht. Entstehung, Gesetzmäßigkeiten und Möglichkeiten zur Vermeidung untersucht an Wiederaufnahmeverfahren im Oberlandesgerichtsbezirk Hamm aus der Zeit von 1950 bis 1958. (Münster: Universität Münster, 1965), 1.

31 Karl Peters. Fehlerquellen im Strafprozess - Eine Untersuchung der Wiederaufnahmeverfahren in der Bundesrepublik Deutschland - Volumen 2: Systematische Untersuchungen und Folgerungen. (Karlsruhe: C. F. Müller, 1972), 1; Karl Peters. Fehlerquellen im Strafprozess - Eine Untersuchung der Wiederaufnahmeverfahren in der Bundesrepublik Deutschland - Volumen 3: Wiederaufnahmerecht". (Karlsruhe: C. F. Müller, 1974), 1.

32 Barbara Dunkel. Fehlentscheidungen inder Justiz. SystematischeAnalysevon Wiederaufnahmeverfahren in Strafverfahren im Hinblick auf Häufigkeit und Risikofaktoren. (Baden-Baden: Nomos, 2018), 179, 190.

33 Fredericke Leuschner, Martin Rettenberger y Axel Dessecker. "Imprisoned But Innocent: Wrongful Convictions and Imprisonments in Germany, 1990-2016", En Crime \& Delinquency, 2019. 


\subsection{Frecuencias en experimentos}

El experimento seguramente más conocido sobre confesiones falsas es el llamado "Computer-Crash-Experiment", que fue inventado por Kassin y Kriechel. ${ }^{34}$ En este caso, el objetivo de la prueba era medir el tiempo de respuesta de los participantes. Para ello, la prueba consistía en no usar una determinada tecla del ordenador bajo ninguna circunstancia. No obstante, el experimento fue manipulado para que la reacción prohibida ocurriera en cualquier momento.

Ante la acusación de haber presionado la tecla prohibida, el 69\% de los 75 participantes firmaron una confesión, a pesar de que no habían hecho nada malo objetivamente.

Varios experimentos demuestran que, en condiciones apropiadas, es probable que una persona acusada haga una confesión falsa, Forrest et al. ${ }^{35}$ (61\%), Redlich y Goodman ${ }^{36}(69 \%)$, Horselenberg et al. ${ }^{37}(82 \%)$, Perillo y Kassin ${ }^{38}(61 \%)$ y Normile y Scherr $^{39}(40,8 \%)$. Sin embargo, estos porcentajes considerablemente altos de confesiones falsas no pueden extrapolarse fácilmente a la vida real, ya que pueden controlar todas las condiciones externas. ${ }^{40}$

\subsection{Frecuencia en encuestas}

Las encuestas son adecuadas para obtener información sobre la frecuencia con la que se realizan confesiones falsas. De esta manera, los afectados también pueden proporcionar información sobre confesiones falsas voluntarias que no fueron

34 Saul Kassin y Katherine Kriechel. "The Social Psychology of False Confessions: Compliance, Internalization, and Confabulation", Psychological Science, $7^{a}$ época, nº 3, (1996): 125.

35 Krista Forrest, Theresa Wadkins y Richard Miller. "The Role of Preexisting Stress on False Confessions: An Empirical Study", Journal of Credibility Assessment and Witness Psychology, $3^{\mathrm{a}}$ época, $\mathrm{n}^{\circ} 1$, (2002): 23.

36 Aluson Reduich y Gail Goodman. "Taking resposinbility for an act not commited. The influence of age and sugessibility", Law and human behavior, $27^{a}$ época, n² 2, (2003): 151.

37 R. Horselenberg, H. Merckelbach y S. Josephs. "Individual differences and false confessions. A conceptual replication of Kassin and Kriechel", Psychology, Crime and Law, nº 9, (2003): 5.

38 Jennifer Perillo y Saul Kassin. "Inside Interrogation: The Lie, The Bluff, and False Confessions", Law and Human Behavior, $\mathrm{n}^{0}$ 35, (2011,): 327.

39 Christopfer Normile y Kyle Scherr. "Police Tactics and Guilt Status Uniquely Influence Suspects' Physiologic Reactivity and Resistance to Confess", Law and Human Behavior, 42ª época, nº 6, (2018): 497.

40 Gisli Gudjonsson. "The Psychology of False Confessions: A Review of the current Evidence", En Police Interrogations and False Confessions. Current Research, Practice, and Policy Recommendations, 1. Edición, editado por Lassiter, Daniel; Meissner, Christian. (Washington D.C.: American Psychological Association, 2010), 43. 
reconocidas por las autoridades policiales. Ahora bien, las encuestas también tienen desventajas: los resultados pueden verse distorsionados por fenómenos como la conveniencia social. ${ }^{41}$ Asimismo, la información proporcionada por los presos, que han hecho una confesión falsa, generalmente no es verificada. En consecuencia, tales estudios solo deben considerarse como una contribución adicional a la visión general de todos los resultados de la investigación. ${ }^{42}$

Los grupos de encuestados más interesantes en relación con las confesiones falsas en los interrogatorios policiales, probablemente sean los detenidos y los policías. Los sujetos de estos grupos han participado en interrogatorios (desde sus respectivas perspectivas) y, por lo tanto, tienen una experiencia relevante. Sin embargo, se les realizaron muchas encuestas a estudiantes. Este grupo tiene la ventaja de que es más accesible y ofrece una visión general de la población. En encuestas con estudiantes, se midió la frecuencia porcentual de aquellas personas que ya habían sido interrogadas por la policía al menos una vez y que habían hecho una confesión falsa. Las encuestas realizadas, principalmente, en los países nórdicos durante los años 2004 a 2009, revelaron que entre el 10 al 25\% de los estudiantes había tenido un interrogatorio policial. En el caso de Gudjonsson et al., ${ }^{43}$ encuestaron a 1.080 estudiantes islandeses, teniendo como resultado que el $25 \%$ de ellos ya habían sido interrogados por la policía, de los cuales el 3,7\% había hecho una confesión falsa. Por otro lado, Steingrimsdottir et al..$^{44}$ encuestaron a 715 estudiantes daneses (10\% / 6,8\%) y Gudjonsson et al. ${ }^{45}$ otros 10.363 estudiantes islandeses $(20 \% / 8,8 \%)$. A su vez, la encuesta más grande hasta el momento, fue realizada por Gudjonsson ${ }^{46}$ en 2009, con 24.627 estudiantes europeos brindando información: $11,5 \%$ y $13,8 \%$.

41 Barbara Dunkel, Fehlentscheidungen in der Justiz. SystematischeAnalysevon Wiederaufnahmeverfahren in Strafverfahren im Hinblick auf Häufigkeit und Risikofaktoren, 345.

42 Kate Houston, Christian Meissner y Jacqueline Evans. "Psychological Processes Underlying True and False Confessions", En Investigative Interviewing, editado por Ray Bull. (New York: Springer, 2014), 21.

43 Gisli Gudjonsson, Jon Sigurdsson, Olafur Bragason, Emil Einarsson y Eva Valdimarsdottir. "Confessions and denials and the relationship with personality", Legal and Criminological Psychology, n 9, (2004): 121.

44 Gunnthora Steingrimsdottir, Hrafnhildur Hreinsdottir, Gisli Gudjonsson, Jon Fridrik Sigurdsson y Thomas NielSEN. "False confessions and the relationship with offending behavior and personality among Danish adolescents", Legal and Criminological Psychology, nº 12, (2007): 287.

45 Gisli Gudjonsson, Jon Sigurdsson, Inga Sigfusdottir y Bjork Asgeirsdottir. "False confessions and individual differences. The importance of victimization among youth", Personality and Individual Differences, $45^{a}$ época, nº 8, (2007): 801.

46 Gisli Gudjonsson, Jon Sigurdsson y Inga Sigfusdottir. "Interrogation and false confessions among adolescents in seven European countries. What background and psychological variables best discriminate between false confessors and non-false confessors?", Psychology, Crime \& Law, n ${ }^{0} 15$, (2009): 711. 


\subsubsection{Encuestas a policías}

Las encuestas realizadas a los agentes de policía para determinar con qué frecuencia se llevan a cabo las confesiones falsas, lamentablemente se han desarrollado de forma limitada. Estos agentes, que tienen una gran experiencia en la materia y que, han realizado innumerables interrogatorios, pueden proporcionar diversa información sobre estas confesiones. No obstante, estos interrogatorios están sujetos a una limitación fundamental: por naturaleza, los agentes solo pueden reportar los casos de confesiones falsas que ellos mismos han reconocido. Esto puede ser particularmente difícil en el caso de una confesion falsa inconsciente, puesto que tales confesiones en algunos casos, se hacen para proteger a una tercera persona. ${ }^{47}$

En una de las encuestas más importantes realizada por Kassin et al., a los agentes de policía en los Estados Unidos, ${ }^{48} 631$ agentes estimaron que el 5\% de los inocentes confesarían. Con este fin, Schell-Leugers y Kassin ${ }^{49}$ llevaron a cabo un estudio de replicación en 2014, en el que fueron entrevistados 63 interrogadores predominantemente europeos, mediante un cuestionario en línea. Los sujetos estimaron que el $12 \%$ de los acusados harían una confesión falsa. Por otro lado, en un estudio muy extenso de Reppucci et al. ${ }^{50}$ en 2010, participaron 1.828 policías estadounidenses. $77 \%$ de los encuestados estimaron que sí habría confesiones falsas (la mayoría: en el 10\% de los casos). Sin embargo, 69\% de los encuestados aún no habían recibido una.

47 Véaseparamásdetalles:ZURNIEDEN, FalscheGeständnisseinderpolizeilichenBeschuldigtenvernehmung. Eine interdisziplinäre Darstellung der theoretischen Grundlagen falscher Geständnisse und zwei empirische Untersuchungen von Polizeibeamten und Inhaftierten zur Häufigkeit und den Gründen von falschen Geständnissen in Deutschland.

48 Saul Kassin, Richard leo, Christian Meissner, Kimberl Richman, Lori Colwell, Amy-May Leach y Dana Fon. "Police Interviewing and Interrogation: A Self-Report Survey of Police Practices and Beliefs", Law and Human Behavior, 31ª época, nº 4, (2007): 392.

49 ennifer Schel-Leugers y Saul Kassin. "Police Practices and Beliefs: A Survey of European Police Investigators", En The Danger of Innocence. Common Sense Beliefs and Misconceptions about False Confessions, editado por Jennifer Schell-Leugers. (Maastricht: Maastricht University, 2014), 51.; Jennifer Schell-Leugers. The Danger of Innocence. Common Sense Beliefs and Misconceptions about False Confessions. (Maastricht: Maastricht University, 2014).

50 Dickon Reppucci, Jessica Meyer y Jessica Kostelnik. "Custodial Interrogation of Juveniles: Results of a National Survey of Police", En Police Interrogations and False Confessions. Current Research, Practice, and Policy Recommendations, 1. Edición, editado por Daniel Lassiter, Christian Meissner. (Washington D.C.: American Psychological Association, 2010), 67. 
En el reciente estudio de zur Nieden (2019) ${ }^{51}$ fueron encuestados 119 policías de cinco Estados federados alemanes y de la Oficina Federal Alemana de Policía Criminal sobre la frecuencia y los antecedentes de las confesiones en los interrogatorios policiales. El 16\% de los funcionarios declararon que habían recibido una confesión falsa al menos una vez en un interrogatorio policial. Zur Nieden no solo se determinó este valor de comparación, sino que también se recolectó el número promedio de confesiones falsas hechas a los agentes de policía: 6,69 confesiones falsas por oficial. También, fue calculada la frecuencia con la que una confesión fue falsa (en relación con todas las confesiones que se hicieron): 1,15\% de todas las confesiones hechas a los funcionarios fueron falsas. Por último, también se determinó la frecuencia con la que se hizo una confesión falsa en un interrogatorio policial: en el 0,35\% de todos los interrogatorios, es decir, uno de cada 283,14 (según los agentes de la policía). ${ }^{52}$ La visión general de los resultados muestra que los agentes de policía se enfrentan a confesiones falsas con mucha más frecuencia de lo que se podría imaginar. Sin embargo, debido a los pocos estudios, existe una mayor necesidad de investigación.

\subsubsection{Encuestas a detenidos}

En este apartado, cabe destacar, las dos encuestas realizadas por Sigurdsson y Gudjonsson a detenidos en 1994 y $1996^{53}$. En ambas, se encontró una frecuencia de confesiones falsas del $12 \%$. Richardson et al. ${ }^{54}$ realizaron encuestas a pacientes adolescentes de psiquiatría forense en Inglaterra en 1991, y encontraron valores significativamente más altos: 23\%. Según la encuesta realizada por Redlich et al.

51 Compárese, también a lo siguiente: ZUR NIEDEN, Falsche Geständnisse in der polizeilichen Beschuldigtenvernehmung. Eine interdisziplinäre Darstellung der theoretischen Grundlagen falscher Geständnisse und zwei empirische Untersuchungen von Polizeibeamten und Inhaftierten zur Häufigkeit und den Gründen von falschen Geständnissen in Deutschland.

52 Este artículo no entrará en el trasfondo lógico de los diferentes números entre los detenidos y la policía. Para más información, véase zUR NIEDEN, Falsche Geständnisse in der polizeilichen Beschuldigtenvernehmung. Eine interdisziplinäre Darstellung der theoretischen Grundlagen falscher Geständnisse und zwei empirische Untersuchungen von Polizeibeamten und Inhaftierten zur Häufigkeit und den Gründen von falschen Geständnissen in Deutschland.

53 Gisul Gudjonsson y Jon Sigundsson. "How frequently do false confessions occur? An empirical study among prison inmates", Psychology, Crime \& Law, n0 1, (1994): 21; Jon Sigurdsson y Gisli Gudjonsson. "The psychological characteristics of 'false confessors'. A study among Icelandic prison inmates and juvenile offenders", Personality and Individual Differences, $20^{a}$ época, nº 3, (1996): 321.

54 G. Richardson, Gisli Gudjonsson y T. Kelly. "Interrogative suggestibility in an adolescent forensic population", en Journal of Adolescence, n 8, (1995): 211. 
en $2010^{55}$ a 1.249 delincuentes con trastornos en su salud mental en los Estados Unidos: 22\%. No obstante, los resultados de Gudjonsson et al. ${ }^{56}$ indican que estos altos porcentajes no solo están relacionados con la salud mental de los sujetos de prueba, ya que se encuestó a un total de 90 detenidos en 2008 y se encontró un valor del $24 \%$ de confesiones falsas.

En Alemania, Volbert et al. ${ }^{57}$ descubrieron en 2016, cuando entrevistaron a 153 pacientes con tratamiento psiquiátrico forense impuesto (Maßregelvollzugspatienten), que el $25 \%$ de ellos ya había hecho una confesión falsa. Por su parte, Gewehr et al. ${ }^{58}$ determinaron valores significativamente más bajos, encuestando a 250 (en su mayoría ex detenidos) sobre sus experiencias en el interrogatorio policial. En este caso, el 4,5\% de los sujetos declararon que habían hecho una confesión falsa al menos una vez.

En el estudio actual de Gubi-Kelm et al. $(2020)^{59}$ solo se entrevistó a prisioneros alemanes, pero, este pequeño número es una excepción. En una muestra de 66 sujetos, 23 detenidos declararon que habían hecho una confesión falsa a la policía al menos una vez en sus vidas. El valor del 35\% determinado aquí ha sido el más alto que se ha encontrado hasta ahora en estudios similares en Alemania.

En su estudio de 2019, zur Nieden encuestó a 351 detenidos alemanes en cinco Estados federados. El 21,7\% de los sujetos declararon que habían hecho una confesión falsa al menos una vez. Pero no solo este valor de comparación fue determinado. También logró recolectar el número promedio de confesiones falsas hechas por cada prisionero: 2,47 confesiones falsas. Adicional a ello, se calculó la frecuencia con la que una confesión fue falsa (en relación con todas las confesiones que se hicieron):

55 Aluison Redich, Alicia Summers y Steven Hoover. "Self-reported false confessions and false guilty pleas among offenders with mental illness", Law and Human Behavior, 34a época, n0 1, (2010): 79.

56 Gisli Gudjonsson, Jon Sigurdsson, O. Bragason, A. Newton y E. Einarsson, "Interrogative suggestibility, compliance and false confessions among prisoners and their relationship with attention deficit hyperactivity disorder (ADHD) symptoms", Psycholgical Medicine, nº 38, (2008): 1040.

57 Volbert, May, Hausam y Lau, “Confessions and Denials When Guilty and Innocent: Forensic Patients' Self-Reported Behavior During Police Interviews" 6.

58 Elsa Gewehr, Lennart May, Yonna Raible, Johannes Zimmermann y Renate Volbert. "Guilty and innocent suspects' perceptions of their police interviews. ... Or why being friendly is a police officers' best shot", En Sesión de póster en la conferencia anual de la Asociación Europea de Psicología del Derecho (EAPL). (Finlandia: Turku, 2018).

59 Silvia Gubi-Kelm, Tuule Grolig, Benjamin Strobel, Simon Ohlig y Alexander Schmidt. "When Do False Accusations Lead to False Confessions? Preliminary Evidence for a Potentially Overlooked Alternative Explanation", en Journal of Forensic Psychology: Research and Practice (todavía inédita), (2020). 
el $11,69 \%$ de todas las confesiones hechas fueron falsas. Finalmente, también fue posible determinar la frecuencia con la que se hizo una confesión falsa en un interrogatorio policial: en el 3,34\% de todos los interrogatorios, es decir, en uno de cada 29,95.

Los resultados de los estudios evidencian que muchos de los detenidos ya han hecho confesiones falsas, incluso si los números difieren mucho, debe quedar claro que este es un problema grave.

Los datos de Volbert et al. y zur Nieden confirmaron que existen confesiones falsas en Alemania. Sin embargo, las cifras que corresponden a los resultados de Gubi-Kelm et al. podrían indicar una cifra más alta. En los resultados de zur Nieden también se puede ver que aproximadamente cada décima confesión realizada en un interrogatorio policial es falsa, y que aproximadamente uno de cada 30 interrogatorios resulta en una confesión falsa (según los detenidos). Estos valores pueden servir como valores comparativos para futuros estudios; y solo queda esperar que dichos valores sean confirmados.

\section{Conclusión}

Las investigaciones internacionales muestran que las confesiones falsas en los interrogatorios policiales se producen en un grado bastante alto.

Por el contrario, no se pueden especificar valores generales sobre su frecuencia. Por un lado, esto se debe a que los estudios siguen diferentes enfoques y, a menudo, no miden valores directamente comparables. Además, algunos de los resultados difieren considerablemente entre sí, por lo que hasta ahora solo se pueden usar como valores de referencia. Hay diferentes razones de cómo surgen estos resultados. Asi, por ejemplo, los estudios se llevaron a cabo en diferentes momentos y en distintos países. De igual manera, los grupos de voluntarios siguen siendo diferentes (por ejemplo, delincuentes "regulares" y delincuentes con trastornos mentales), a pesar de tener una categoría superior común, que es la de detenidos.

Por otro lado, las diversas formas de estudios tienen desventajas individuales que hacen imposible recopilar todas las confesiones falsas: en el caso del análisis de archivos, solo se pueden descubrir las confesiones falsas que también se conocen. Del mismo modo, los agentes de policía solo pueden reportar las confesiones falsas que han reconocido como tales. Por último, los detenidos no saben nada de confesiones erróneamente hechas por ignorancia y no quieren reportar ninguna confesión falsa que hayan hecho voluntariamente. 
Esto muestra que la frecuencia de las confesiones falsas solo se puede aproximar. Por esta razón, se deben seguir varios enfoques en el futuro, con análisis de archivos y encuestas a las personas más adecuadas. Además, tendría sentido este último aspecto si los futuros estudios recolectaran los datos comparables de todas las maneras posibles.

Debe enfatizarse positivamente que el tema de las confesiones falsas también se está convirtiendo cada vez más en el foco de la investigación en Alemania. Recientemente, se han llevado a cabo nuevos estudios, especialmente con los acusados. Sin embargo, hay un déficit con respecto a la perspectiva policial, debido a que hay significativamente menos investigación disponible en Alemania e internacionalmente. Más concretamente, son estos últimos, los encargados de realizar los interrogatorios y quienes pueden evitar confesiones falsas mediante un enfoque prudente y capacitado, por lo que se necesita una mayor investigación en esta área.

En este sentido, gracias a los dos estudios que han realizado en 2019 en Alemania, fue posible comparar por primera vez los dos importantes grupos de sujetos de prueba, los agentes de policía y los prisioneros. Estos datos aseguran que el 11,69\% de todas las confesiones hechas por los detenidos eran falsas, y el 1,15\% hechas a los agentes de policía el Según la información de ambos grupos, 2,19\% de todas las confesiones fueron falsas. Según el autor, la diferencia entre los datos de estos dos grupos de sujetos muestra el campo oscuro. Por lo tanto, solo cada décima (intecionalmente) confesión falsa recibida por agentes de policía fue reconocida como tal. ${ }^{60}$ Incluso está por verse si una investigación adicional confirmará estos valores, teniendo en cuenta que los agentes de policía solo reconocen una fracción de las confesiones incorrectas. Esto resalta la necesidad de poner mayor énfasis en este tema, particularmente en la capacitación de los interrogadores.

\section{Bibliografía}

Bedau, Hugo y Michael Radelet. "Miscarriages of Justice in Potentially Capital Cases", Stanford Law Review, nº 40, (1987). 
Connors, Edward, Thomas lundregan, Neal Miller y Tom McEwen. Convicted by Juries,

Exonerated by Science. Case Studies in the Use of DNA Evidence to Establish Innocence After Trial, Departamento de Justicia de los EE.UU. Alejandría: Oficina de Programas de Justicia, Instituto Nacional de Justicia, 1996.

Dunkel, Barbara. Fehlentscheidungen in der Justiz. Systematische Analyse von Wiederaufnahmeverfahren in Strafverfahren im Hinblick auf Häufigkeit und Risikofaktoren. Baden-Baden: Nomos, 2018.

Forrest, Krista, Theresa Wadkins y Richard Miller. The Role of Preexisting Stress on False Confessions: An Empirical Study, Journal of Credibility Assessment and Witness Psychology, $3^{a}$ época, $n^{0} 1,(2002)$.

Gewehr, Elsa, Lennart May, Yonna Raible, Johannes Zimmermann y Renate Volbert. "Guilty and innocent suspects' perceptions of their police interviews. ...Or why being friendly is a police officers' best shot", Sesión de póster en la conferencia anual de la Asociación Europea de Psicología del Derecho (EAPL). Finlandia: Turku, 2018.

Gross, Samuel, Kristen Jacoby, Daniel Matheson, Nicholas Montgomery y Sujata Patil. "Exonerations in the United States 1989 through 2003", Journal of Criminal Law and Criminology, 95a época, $\mathrm{n}^{0} 2$ 2, (2005).

Gubi-Kelm, Silvia, Tuule Grolig, Benjamin Strobel, Simon Ohlig y Alexander Schmidt. “When Do False Accusations Lead to False Confessions? Preliminary Evidence for a Potentially Overlooked Alternative Explanation", Journal of Forensic Psychology: Research and Practice (todavía inédita), (2020).

Gudjonsson, GisLI y Jon Sigurdsson. "How frequently do false confessions occur? An empirical study among prison inmates", Psychology, Crime \& Law, n0 1, (1994).

GudJonsSON, GISLI, Jon IGURDSSON Y Inga SigfusdotTiR. "Interrogation and false confessions among adolescents in seven European countries. What background and psychological variables best discriminate between false confessors and nonfalse confessors?", Psychology, Crime \& Law, n 15, (2009).

Gudjonsson, Gisli, Jon Sigurdsson, Inga Sigfusdottir y Bjork Asgeirsdottir. "False confessions and individual differences. The importance of victimization among youth", Personality and Individual Differences, $45^{a}$ época, nº 8, (2007).

Gudjonsson, Gisli, Jon Sigurdsson, Olafur Bragason, Emil Einarsson y Eva Valdimarsdottir.

"Confessions and denials and the relationship with personality", Legal and Criminological Psychology, no 9, (2004).

Gudjonsson, Gisl. "The Psychology of False Confessions: A Review of the current 
Evidence", En Police Interrogations and False Confessions. Current Research, Practice, and Policy Recommendations, 1. Edición, editado por Lassiter, Daniel; Meissner, Christian. Washington, D.C.: American Psychological Association, 2010.

Gudjonsson, Jon Gisl, Sigurdsson, O. Bragason, A.Newton, E. Einarsson. "Interrogative suggestibility, compliance and false confessions among prisoners and their relationship with attention deficit hyperactivity disorder (ADHD) symptoms", Psycholgical Medicine, $\mathrm{n}^{0}$ 38, (2008).

Horselenberg, R., H. Merckelbach y S. Josephs. "Individual differences and false confessions. A conceptual replication of Kassin and Kriechel", Psychology, Crime and Law, $n^{0}$ 9, (2003).

Houston, Kate, Christian Meissner y Jacoueline Evans. "Psychological Processes Underlying True and False Confessions", En Investigative Interviewing, editado por Bull, Ray. New York: Springer, 2014.

Kassin, Saul y Gisl Gudjonsson. "The Psychology of Confessions. A Review of the Literature and Issues", Psychological Science in the Public Interest, $5^{\mathrm{a}}$ época, $n^{0} 2$, (2004).

Kassin, Saul y Katherine Kriechel. "The Social Psychology of False Confessions: Compliance, Internalization, and Confabulation", Psychological Science, $7^{\text {a }}$ época, nº 3, (1996).

Kassin, Saul y Lawrence Wrightsman, , "Confession Evidence", en Kassin, Saul; Wrightsman, Lawrence (editores), The Psychology of Evidence and Trial Procedure, Beverly Hills, Sage, 1985.

Kassin, Saul. "The social psychology of false confessions", Social Issues and Policy Review, ga época, $\mathrm{n}^{0}$ 1, (2015).

Kassin, Saul. Richard Leo, Christian Meissner, Kimberl Richman, Lori Colwell, Amy-May Leach y Dana Fon. "Police Interviewing and Interrogation: A Self-Report Survey of Police Practices and Beliefs", Law and Human Behavior, $31^{\mathrm{a}}$ época, $\mathrm{n}^{0} 4$, (2007).

KIwit, Walter. Fehlurteile im Strafrecht. Entstehung, Gesetzmäßigkeiten und Möglichkeiten zur Vermeidung untersucht an Wiederaufnahmeverfahren im Oberlandesgerichtsbezirk Hamm aus der Zeit von 1950 bis 1958. Münster: Universität Münster, 1965. 
Lange, Regina. Fehlerquellen im Ermittlungsverfahren. Eine Auswertung von 1110 Wiederaufnahmeverfahren. Heidelberg: Kriminalistik, 1980.

Leuschner, Fredericke, Martin Rettenberger y Axel Dessecker. "Imprisoned But Innocent: Wrongful Convictions and Imprisonments in Germany, 1990-2016", Crime \& Delinquency, 2019.

Normile, Christopfer y Kyle Scherr. "Police Tactics and Guilt Status Uniquely Influence

Suspects' Physiologic Reactivity and Resistance to Confess", Law and Human Behavior, 42ª época, $\mathrm{n}^{0}$ 6, (2018).

ÖHNER, VRÄ̈̈TH. "Unabweisbare Gewissheit. Zur Kritik des Geständnisses", En Das Geständnis und seine Instanzen: Zur Bedeutungsverschiebung des Geständnisses im Prozess der Moderne, editado por Engberg-Pedersen, Anders; Huffmaster, Michael; Nordhausen, Eric; Öhner, Vrääth. Berlin: Turia + Kant, 2011.

Oxburgh, Gavin, Ivar Fahsing, Kate Haworth Y Pete Blair. "Interviewing Suspected Offenders", En Communication in Investigative and Legal Contexts: Integrated Approaches from Forensic Psychology, Linguistics and Law Enforcement, 1. edición, editado por Oxburgh, Gavin; Myklebust, Trond; Grant, Tim; Milne, Rebecca. Chichster: Wiley-Blackwell, 2015.

Perillo, Jennifer y Saul Kassin. "Inside Interrogation: The Lie, The Bluff, and False Confessions", Law and Human Behavior, n0 35, (2011).

Peters, Karl. Fehlerquellen im Strafprozess - Eine Untersuchung der Wiederaufnahmeverfahren in der Bundesrepublik Deutschland - Volumen 3: Wiederaufnahmerecht. Karlsruhe: C. F. Müller, 1974.

Peters, Karl. Fehlerquellen im Strafprozess - Eine Untersuchung der Wiederaufnahmeverfahren in der Bundesrepublik Deutschland - Volumen 2: Systematische Untersuchungen und Folgerungen. Karlsruhe: C. F. Müller, 1972.

Rattner, Arve. "Convicted but innocent: Wrongful conviction and the criminal justice system", Law and Human Behavior, 12ª época, $n^{0} 3$, (1988).

Redlich, Alusison y Gail Goodman. "Taking resposinbility for an act not commited. The influence of age and sugessibility", Law and human behavior, $27^{\mathrm{a}}$ época, $\mathrm{n}^{0} 2$, (2003).

Redlich, Allison, Alicia Summers y Steven Hoover. "Self-reported false confessions and false guilty pleas among offenders with mental illness", Law and Human Behavior, 34a época, nº 1, (2010).

Reppucci, Dickon, Jessica Meyer y Jessica Kostelnik. "Custodial Interrogation of Juveniles: Results of a National Survey of Police", En Police Interrogations and 
False Confessions. Current Research, Practice, and Policy Recommendations, 1. edición, editado por Lassiter, Daniel; Meissner, Christian. Washington D.C.: American Psychological Association, 2010.

Richardson, G., Gisıl Gudjonsson Y T. Kelly. "Interrogative suggestibility in an adolescent forensic population", Journal of Adolescence, n 8 , (1995).

Scheck, Barry, Peter Neufeld y Jim Dwyer. Actual Innocence. New York: Doubleday, 2000.

Schell-Leugers, Jennifer y Saul Kassin. "Police Practices and Beliefs: A Survey of European Police Investigators", En The Danger of Innocence. Common Sense Beliefs and Misconceptions about False Confessions, editado por SchellLeugers, Jennifer. Maastricht: Maastricht University, 2014.

Schell-Leugers, Jennifer. The Danger of Innocence. Common Sense Beliefs and Misconceptions about False Confessions. Maastricht: Maastricht University, 2014.

Schramm, Edward. "Das Geständnis im Deutschen Strafprozessen EngbergPedersen", En Das Geständnis und seine Instanzen: Zur Bedeutungsverschiebung des Geständnisses im Prozess der Moderne, editado por Anders; Huffmaster, Michael; Nordhausen, Eric; Öhner, Vrääth. Berlin: Turia + Kant, 2011.

SIGURDSSON, Jon y GISLI GudJonsson. "The psychological characteristics of 'false confessors'. A study among Icelandic prison inmates and juvenile offenders", Personality and Individual Differences, 20ª́poca, n 3, (1996).

Steingrimsdottir, Gunnthora, Hrafnhildur Hreinsdottir, Gisli Gudjonsson, Jon Fridrik Sigurdsson y Thomas Nielsen. "False confessions and the relationship with offending behavior and personality among Danish adolescents", en Legal and Criminological Psychology, $\mathrm{n}^{0}$ 12, (2007).

Volbert, Renate y Claudia Böhm. "Falsche Geständnisseen", En Handbuch der Rechtspsychologie, editado por Volbert, Renate; Steller, Max. Göttingen: Hgrefe, 2008.

Volbert, Renate, "Falsche Geständnisse bei Kapitaldelikten. Grundlagen", En Festschrift für Ulrich Eisenberg zum 70. Geburtstag, editado por Müller, Henning; Sander, Günther; Válková, Helena. München: Beck, 2009.

Volbert, Renate, Lennart May, Joachim Hausam y Steffen Lau. "Confessions and Denials When Guilty and Innocent: Forensic Patients' Self-Reported Behavior During Police Interviews", Frontiers in Psychiarty, 10ª́poca, n0 168, (2019). 
Wrightsman, Lawrence y Saul Kassin. Confessions in the Courtroom. London: Sage, 1993

Zur Nieden, Valentin. "Falsche Geständnisse in der polizeilichen Beschuldigtenvernehmung. Eine interdisziplinäre Darstellung der theoretischen Grundlagen falscher Geständnisse und zwei empirische Untersuchungen von Polizeibeamten und Inhaftierten zur Häufigkeit und den Gründen von falschen Geständnissen in Deutschland". Tesis doctoral todavía inédita, Universidad de Hamburgo, 2020. 